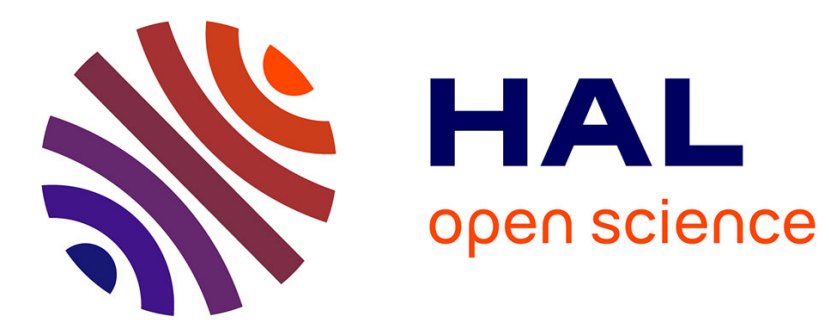

\title{
Automatic closed edge detection using level lines selection
}

\author{
Thomas Hurtut, Farida Cheriet
}

\section{To cite this version:}

Thomas Hurtut, Farida Cheriet. Automatic closed edge detection using level lines selection. International Conference on Image Analysis and Recognition, 2007, Canada. pp.187-197. hal-00430458

\section{HAL Id: hal-00430458 \\ https://hal.science/hal-00430458}

Submitted on 8 Nov 2009

HAL is a multi-disciplinary open access archive for the deposit and dissemination of scientific research documents, whether they are published or not. The documents may come from teaching and research institutions in France or abroad, or from public or private research centers.
L'archive ouverte pluridisciplinaire HAL, est destinée au dépôt et à la diffusion de documents scientifiques de niveau recherche, publiés ou non, émanant des établissements d'enseignement et de recherche français ou étrangers, des laboratoires publics ou privés. 


\title{
Automatic closed edge detection using level lines selection
}

\author{
Thomas Hurtut ${ }^{1,2}$ and Farida Cheriet $^{1}$ \\ 1 Ecole Polytechnique de Montreal, LIV4D, Montreal, Canada \\ 2 Ecole Nationale Superieure des Telecommunications, TSI, Paris, France
}

\begin{abstract}
This paper presents a closed edge detection method based on a level lines selection approach. The proposed method is based on an unsupervised probabilistic scheme using an a contrario method. A level line is considered meaningful if its contrast and length is unlikely to be due to chance. Besides being unsupervised, this method exploits a tree structure. The first step of the proposed approach is to reduce the meaningful level lines set using this hierarchical structure. Compared with a previous method using the same principle, our method achieve a $67 \%$ reduction rate of irrelevant levels lines. The second step of the proposed approach illustrates the high flexibility of using closed edge boundaries such as levels lines. Using a rather simple curvature analysis, the proposed method detects anatomical structures boundaries from CT scan images.
\end{abstract}

\section{Introduction}

Edge detection challenges the vision community since its beginnings. Many solutions have been proposed. So called classic methods are based on gradient detection by filters [1], masks, Laplacian zero-crossing [2,3] and adaptive masks [4]. Analytic approaches follows, among them are Canny [5] and Deriche [6] detectors. Unfortunately, they require fine and supervised parameters tuning and are also sensitive to image noise. They also consider edges as a local concept which is opposed to Gestalt laws [7]. One of the biggest drawback of these methods is their open edge processing. This require a difficult and hazardous edge closing step [8-10]. Besides these methods, Kass et al. [11,12] introduced the active contour model or snake. These semi-automatic methods use a deformable model which minimizes an internal deformation energy subject to external forces derived from images. Closely, level sets approaches allows topology flexibility [13,14]. Here, so-called level sets are defined as the zeros levels of surfaces in a three dimensions space. The active contour model is also close to the geodesic model [15]. These three kind of deformable methods also require many parameters to be tuned, and are strongly dependent on the initialization.

Mathematic morphology proposed to use another kind of image level sets to extract object contours. These level sets are different from the ones previously defined but unfortunately hold the same name. Given an image $u$, a level set at the value $\lambda$ is $\chi_{\lambda}(u)=\left\{x \in \mathrm{R}^{2}, u(x) \geq \lambda\right\}$. One important property of this 
representation is that no information is lost, since we can reconstruct an image from the whole family of its level sets [16]. It thus provides a complete representation of images. It is also worth noting how large shapes are already present with as few as 5 or 6 coarsely quantized levels. Levels sets boundaries are called level lines, and the whole family of the level lines of an image is called the topographic map [17]. This representation enjoys several important advantages. It is contrast invariant. Levels lines are closed curves (except the ones meeting the image borders). It is a hierarchical representation. An important property is that object contours locally coincide with level lines [16]. Unfortunately, the topographic map contains also the texture details and noise effects. Therefore Desolneux et al. presented a level lines selection method [18], which has been compared with active contour model [19]. Desolneux et al. do not claim to propose a ready to use optimal edge detector. Instead they consider their method only provide a set of feasible, i.e. acceptable edges.

Indeed the resulting set of boundaries is not optimal. As we shall see it contains too much irrelevant boundaries to be used directly as an object boundaries detector. Two contributions are proposed in this paper. The first one uses the hierarchical representation of the boundaries set to reduce the number of irrelevant boundaries and aims all kind of images. The second one is designed for CT scan images which handle some characteristics that require special methods as it often occurs in medical image analysis [20]. Indeed in the context of deformable organ modeling from volumetric data such as CT scan images, in order to simulate treatment, it is useful to detect only the boundaries of the main anatomical structures such as fat, muscles and bones. Therefore, the objective of the second contribution is to detect only relevant boundaries that satisfy some specific criteria defined with respect to some descriptors of the targeted structures. Furthermore the second contribution allows to eliminate some specific noise in CT scan images.

The plan of the paper is as follows. We shall give more details on Desolneux et al. probabilistic scheme at the section 2.1 followed by our contributions presented at the section 2.2 and 2.3. It consists on a recursive course of the level lines tree structure on homogeneous subtrees, using of a topological criterion between included shapes, and a curvature analysis. These contributions reduce and optimize the final boundaries set. We present one medical application of this method using CT scan images at the section 3 .

\section{Proposed method}

Desolneux et al. recently introduced a parameter-free method to detect feasible edges in a digital image without any a priori information [18], only using a basic perceptual principle called Helmholtz principle. It affirms that an observed geometric structure is perceptually meaningful if its number of occurrences is very small in a random situation. This principle has been successfully applied to numerous vision problems such as alignments detection [21], vanishing point 
detection [22], histogram modes analysis [23], shape indexation [24], and spatialcolor image retrieval [25].

\subsection{Probabilistic scheme}

The proposed method is based on the probabilistic scheme used in $[18,19]$. A geometric event is said to be $\varepsilon$-meaningful if this event occurs less than $\varepsilon$ times in a random situation. This is an application of the general principle of a contrario methods: we do perceive events that are unlikely in a white noise situation. Here, the geometric event is the presence of long enough and contrasted enough level line. Let $u$ be a gray level image. We search the level lines of $u$ along which contrast assumes unexpectedly high values. These values are compared to the distribution of contrast following the law of the random variable $X$ :

$$
\forall \mu>0, P(X>\mu)=\frac{\#\{x,|\nabla u|>\mu\}}{\#\{x,|\nabla u|>0\}}
$$

We denote by $H(\mu)$ this empirical probability, and let $n_{u}$ be the number of level lines of $u$. According to Desolneux et al. definition, a level line $C$ is $\varepsilon-$ meaningful if

$$
N F A(C)=n_{u} H\left(\min _{x \in C}|\nabla u(x)|\right)^{l / 2}<\varepsilon
$$

where $l$ is the length of $C$. This number is called number of false alarms (NFA). It measures the meaningfulness of an event, and it depends only on the length of $C$ and its minimal contrast. Low NFAs indicate perceptually meaningful contrasted boundaries. This event definition is discussed in [19]. It is worth noting that this selection method only depends on one parameter: $\varepsilon$. Practically, this parameter is very robust. Desolneux et al. show that it is logarithmically dependent and thus varies very slowly. Therefore it is always fixed to $\varepsilon=1$ whatever the image and source are. Hence the method is said to be parameterless. At this point, the method selection can be summarized as keeping all the level lines that have a NFA inferior to $\varepsilon=1$.

The hierarchical structure of the level lines representation is preserved beyond this step. Two meaningful level lines are either disjoint or linked by inclusion. The meaningful level lines set is represented by a tree structure where each node contain one level line. A level line is closed or open (if it crosses the image border). Depending on the gradient sign along the level line, it also said to be positive or negative. An example of a synthetic image and its resulting level lines tree structure is presented on figure 1. A positive level line is represented by a white node. Inclusion is bottom-up. A child level line is included in its parent.

The $\varepsilon$-meaningful level lines set is much smaller than the whole topographic map, but still contain a lot of redundant lines. Digital sampled images present thick edges where many parallel level lines take place. Therefore Desolneux et al. define maximal $\varepsilon$-meaningful level lines. A maximal monotone section of a level line tree is a branch of the tree where any node has only one son, the gray level is monotone in this part (nodes are either positive or negative), and is not 


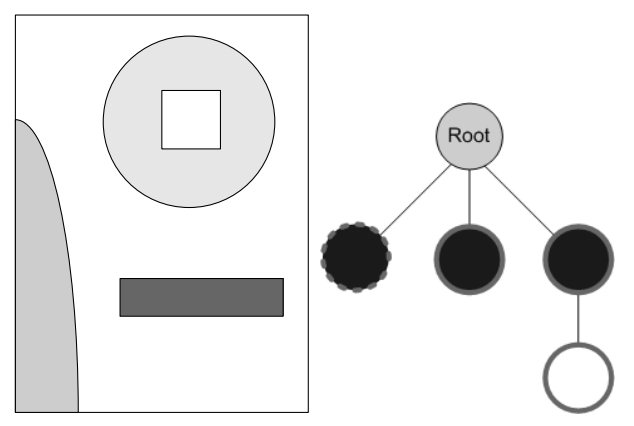

Fig. 1. A synthetic image (left) and its resulting level lines tree (right). Black nodes (resp. white) represent negative line (resp. positive). The doted line represents an open line, crossing the image border

strictly contained in another monotone section. Then a meaningful level line is maximal if it has a minimal NFA in a maximal monotone section. This selection method is presented on figure 2. The branch maximality principle of Desolneux et al. still miss some redundant lines due to its restricted application to maximal monotone section. Two examples of CT Scans image segmentations with this method are showed on figure 3 .

\subsection{Recursive maximality principle on monotone subtrees}

We propose to change the maximality principle by first using a topological criterion. We observed that redundant level lines are close to one parent line or one child line. With sampled lines, this closeness is represented by the fact that no local point of child line $C_{c}$ is very far from a parent line $C_{p}$. The asymmetrical Hausdorff distance $d_{H}$ represents well this kind of proximity:

$$
d_{H}\left(C_{c}, C_{p}\right)=\max _{P_{e} \in C_{c}}\left(\min _{P_{p} \in C_{p}} d\left(P_{c}, P_{p}\right)\right)
$$

where $d\left(P_{e}, P_{p}\right)$ is the euclidean distance between two points. The distance $d_{H}$ is used in our maximality principle.

We also improve the maximality principle by applying it to maximal monotone subtrees. We observed that missed redundant lines are often placed on different tree levels of a same monotone subtree. This happens when at least two close objects are included in a same level line. An example of this situation is shown on figure 4a). Redundancies is also due to texture effects, when at least two small contrasted level lines break the branch into at least two branches. An example of this effect is visible on figure $4 \mathrm{~b}$ ).

Therefore we propose a recursive course applied on monotone maximal subtrees and not only single-parent monotone branch. For every maximal monotone subtree $S$ of the level lines meaningful tree, we recursively search the line $C_{m}$ having the minimal NFA among the subtree. Then, we look upward and downward 


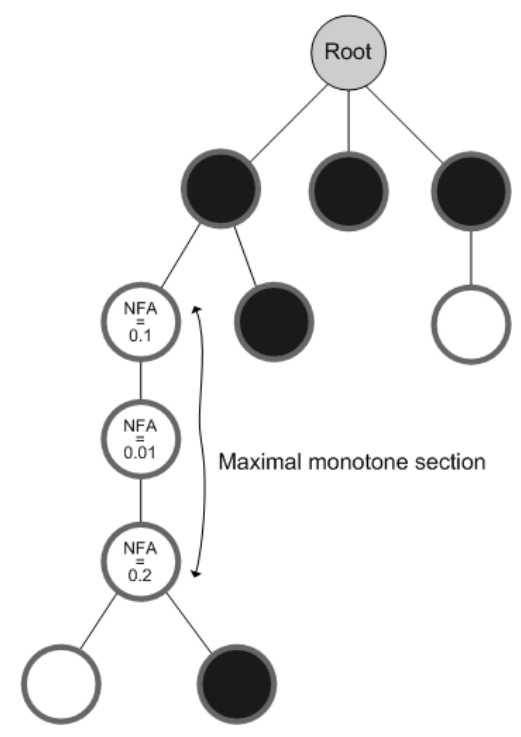

Fig. 2. Branch maximality principle of Desolneux et al [18]. Maximal monotone section of a level line tree is a branch where any node has only one son, the gray level is monotone in this part (nodes are either positive or negative), and is not strictly contained in another monotone section. A meaningful level line is maximal if it has a minimal NFA in a maximal monotone section.

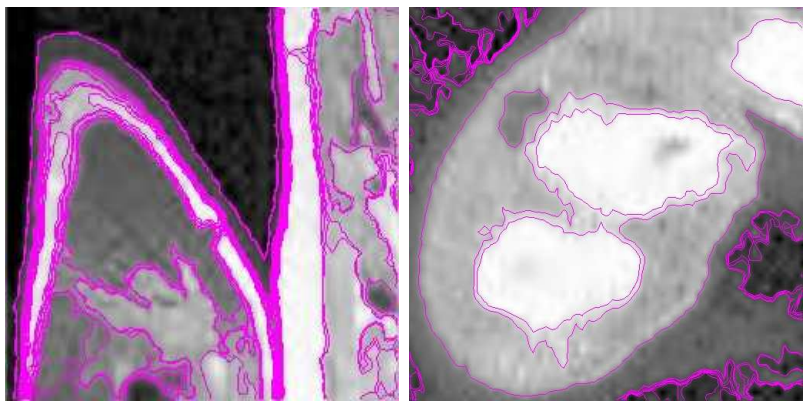

Fig. 3. Two examples using Desolneux et al. branch maximality principle. Left, one thorax MRI. Right, a CT Scan image of the trunk. 


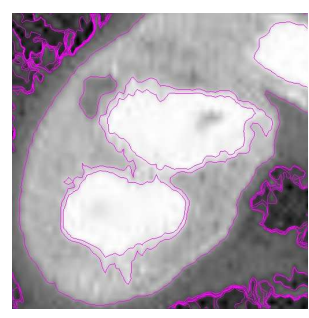

a)

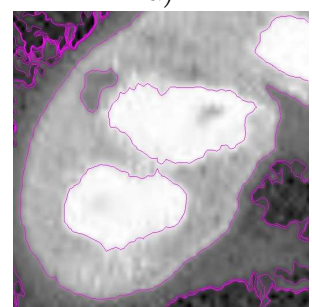

c)

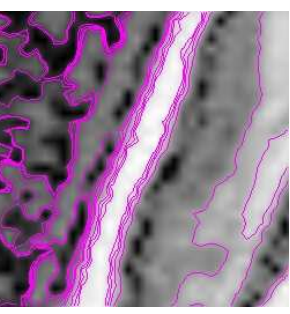

b)

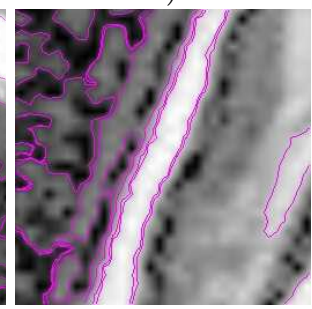

d)

Fig. 4. Oversegmented CT scan image of the trunk and MRI image of the thorax. Up, two examples segmented with Desolneux et al. maximality principle. Bottom, the same examples segmented with our maximality principle.

in the subtree to check if no close line has been selected previously. Closeness is here defined by a threshold on $d_{H}$. If so, the line is selected and removed from the subtree. If one close line is find upward or downward, all the lines on the tree path linking these two lines are removed from the subtree. We then recursively look forward the next minimal NFA in the subtree. The algorithm stops exploring the subtree when it is empty. On figure 1, this algorithm is clarified. The distance threshold $h$ is linked to edge thickness on image. It can be empirically fixed for every image source. Typical values are $h<5$ pixels.

Up to now, the algorithm is very general and can be applied to detect all meaningful objects contours in an image. An important advantage of this method is that it manipulates closed and sampled contours - the level lines. Hence can we easily improve the detection of specific objects or features by adding criteria on curvature, length, contrast sign, etc.

\subsection{Curvature analysis on segmented CT scan images}

CT scan images have a specific important noise. It is visible on Figure 5. This noise is called quantum mottle and is due to the fact that an X-ray beam is an uneven source of X-rays photons [26]. This uneven source has an effect on the film image via the fluorescent screen. The quantum mottle noise is scattered and missed by the detector. Bodies texture also sometimes leads the proposed method in selecting many boundaries which are not relevant regarding our application. Many denoising solutions have been proposed in the literature e.g. using wavelets 


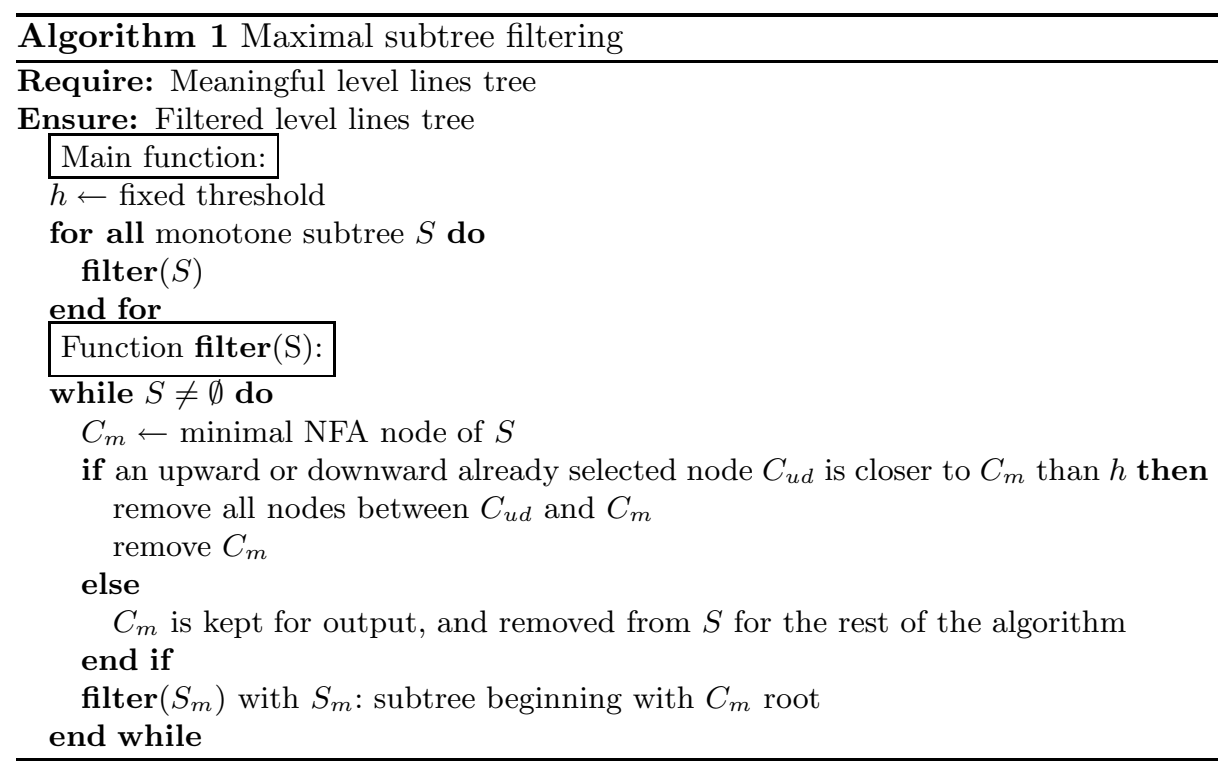

[27], adaptive scale space [28], Wiener filtering [29] or air gap placement on the imaging plate [30].

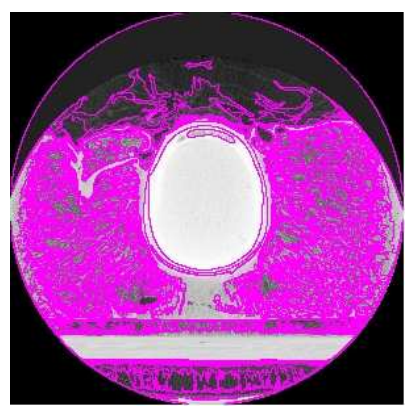

Fig. 5. Quantum mottle noise effect. The X-ray source is uneven and so is the visible image reflected on the fluorescent screen and film during CT scanning.

This geometric noise occurs at a specific granularity scale (related the X-ray beam). This explains why proposed solutions in the literature uses wavelets. We propose to use our level lines set. To eliminate noisy boundaries, we propose a method relying on a two curvature thresholds. Curvature is easily calculated along the selected boundaries: $c_{i}=\left(\theta\left(P_{i-1}, P_{i}\right)-\theta\left(P_{i}, P_{i+1}\right)\right) /\left(P_{i-1} P_{i}-P_{i} P_{i+1}\right)$ on every point $P_{i}$. Curvature is seen as the discrete differential of tangent angle divided by the sampling rate which is fixed and constant for all images and all 
boundaries. For every boundary $\mathcal{B}$ we measure the standard deviation $\sigma_{\mathcal{B}}$ on its whole length, and also the standard deviation $\sigma_{\mathcal{B}}^{L}$ on the partial length $L$ where the standard deviation is maximized. Typically we take $L=10 \%$ of the image diagonal. Our goal here is to detect the boundaries which have at least $L$ part that have a noisy geometrical structure hence high standard deviations.

We manually analyzed relevant boundaries of 5 CT scans to fix these thresholds. The relevant and non relevant boundaries from this set composed of 896 boundaries are shown on Figure 6. Besides a few outlayers, the thresholds $\sigma^{L}<$ 0.45 and $\sigma<0.35$ achieve best results (see Section 3 ). The diagonal alignment on this figure is due to boundaries whose length is smaller than $L$.

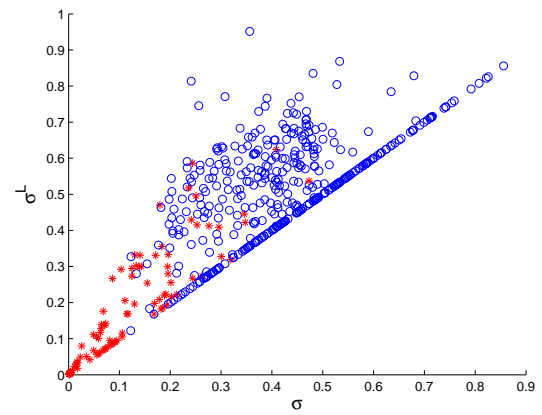

Fig. 6. Partial curvature standard deviation $\sigma^{L}$ graph of relevant and irrelevant boundaries according to the standard deviation $\sigma$. Circle are irrelevant boundaries, and stars are relevant boundaries.

\section{Results}

Experiments have been done on a set of $20 \mathrm{CT}$ scan images from the Human Visible Project [31]. In the following experiments we always used $h=5$. This value is linked to edge thickness on image and can be empirically fixed for every image source.

Comparison results with Canny-Deriche open edge detector [6] and the closededge selection method using the maximal monotone selection principle from Desolneux et al. [18] are shown on Figure 7 from first row to fourth row. The Canny-Deriche detector produces many open edges or misses some contours. An edge closing step remains hazardous in this situation. The average size reduction rate from the boundaries set obtained with the branch maximality principle and the set obtained with the recursive subtrees maximality principle is $67 \%$. This set reduction is not visually obvious because most of the boundaries which have 
been eliminated are multiple very close (inside the $h=5$ pixels distance threshold) boundaries. On all our experiments this reduction of the set size is lossless, meaning absolutely no relevant boundaries are lost.

With the curvature thresholds the reduction raises to $93 \%$. It is visible on the second row of Figure 8 that this $24 \%$ improvement focuses on noisy and texture boundaries. Resulting boundaries meet the objective of the second contribution, that is only detect the main anatomical structures boundaries. Thanks to the flexibility of the closed edge representation, these results are achieved with a quite simple curvature analysis. Future work will include more sophisticated pattern recognition criteria with respect to some descriptors characterizing the targeted structures. Further work is undertaken to demonstrate the clinical relevance of the proposed method by validating the detected boundaries by an expert on actual data of patients.

\section{Conclusion}

In this paper we propose an automatic unsupervised edge detector inspired from the Desolneux et al. level lines selection approach. We propose a new maximality principle and apply it to maximal monotone subtrees. During our experiments, it performed a detected boundaries set reduction of $67 \%$. We also illustrate the high flexibility of this kind of approach addressing an important CT scans image segmentation problem, by using simple and intuitive criteria on level lines such as a two thresholds curvature analysis. In the situation of our application this improvement raised the size reduction of the set to $93 \%$. Our goal is to show the interest of this approach to medical imaging. It is very robust to noise effect, contrast invariant [17], parameter-less (besides the topological criterion), very flexible and ready-to-use as a pattern recognition preprocessing. It provides closed contours easy to handle for various medical applications, and it doesn't require any shape initialization like active contour model.

\section{References}

1. V. Torre and T. Poggio, "On edge detection," IEEE Trans. on Pattern Analysis and Machine Intelligence, vol. 8, pp. 147-153, 1986.

2. D. Marr and E. Hildreth, "Thoery of edge detection," Proc. Rpyal Soc. London, vol. 207-B, pp. 187-217, 1980.

3. R. Haralick, "Digital step edges from zero crossing of second directionnal derivatives," IEEE Trans. on Pattern Analysis and Machine Intelligence, vol. 6, pp. 58$68,1984$.

4. M. Hueckel, "An operator which locates edges in digitized pictures," J. Ass. Comp. Mach., vol. 18, pp. 113-125, 1971.

5. J. Canny, "A computationnal approach to edge detection," IEEE Trans. on Pattern Analysis and Machine Intelligence, vol. 8, pp. 679-698, 1986.

6. R. Deriche, "Using canny's criteria to derive recursively implemented optimal edge detector," Int. J. Computer Vision, vol. 1, pp. 167-187, 1987.

7. G. Kanizsa, La grammaire du Voir. Diderot, 1996. 

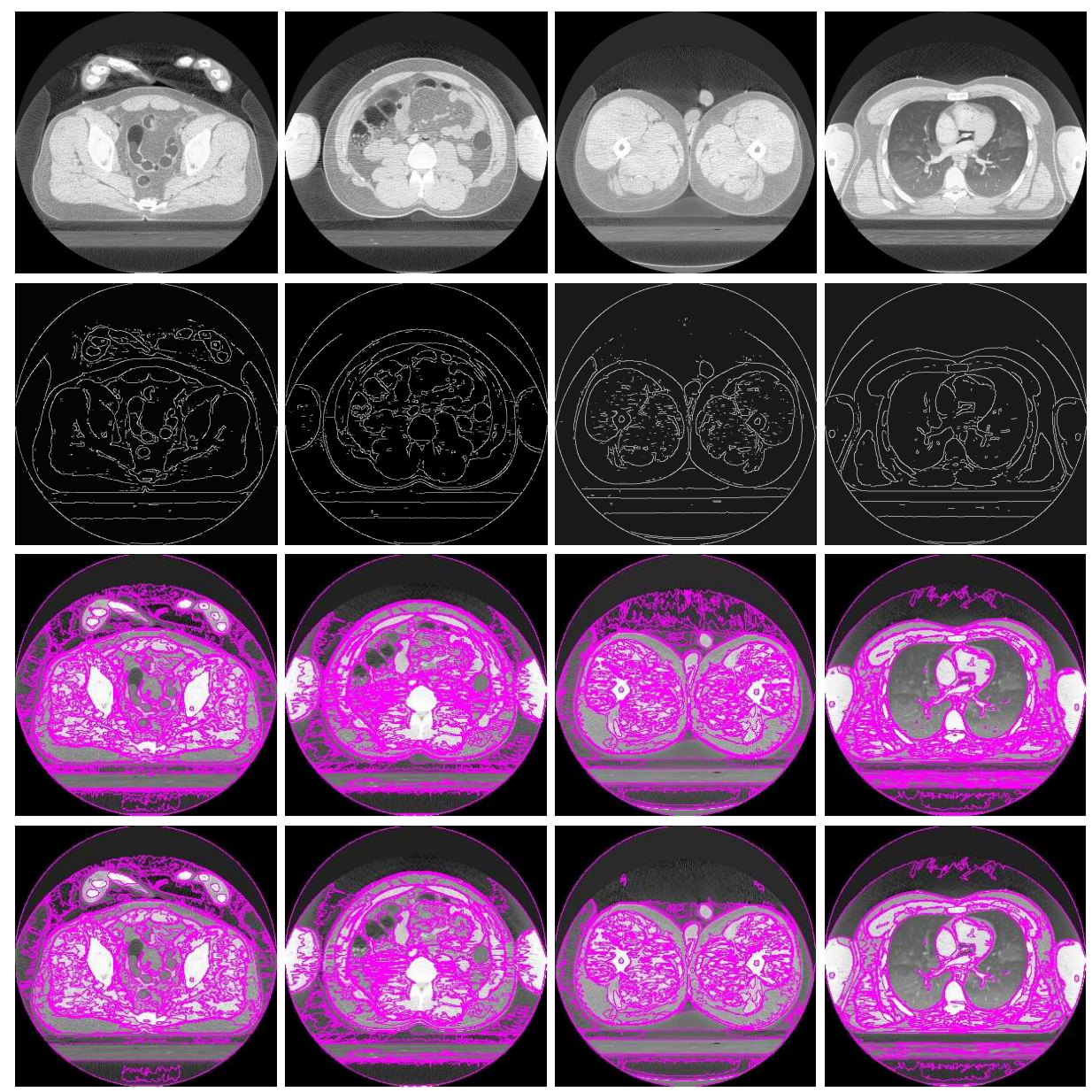

Fig. 7. First row: original CT-Scan. Second row: Canny-Deriche open edge detection. Third row: closed edge detection with the maximal monotone selection principle. Fourth row: closed edge detection with the proposed maximality selection principle without the curvature analysis. The average size reduction rate from the boundaries set obtained with the branch maximality principle and the set obtained with the recursive subtrees maximality principle is $67 \%$. 

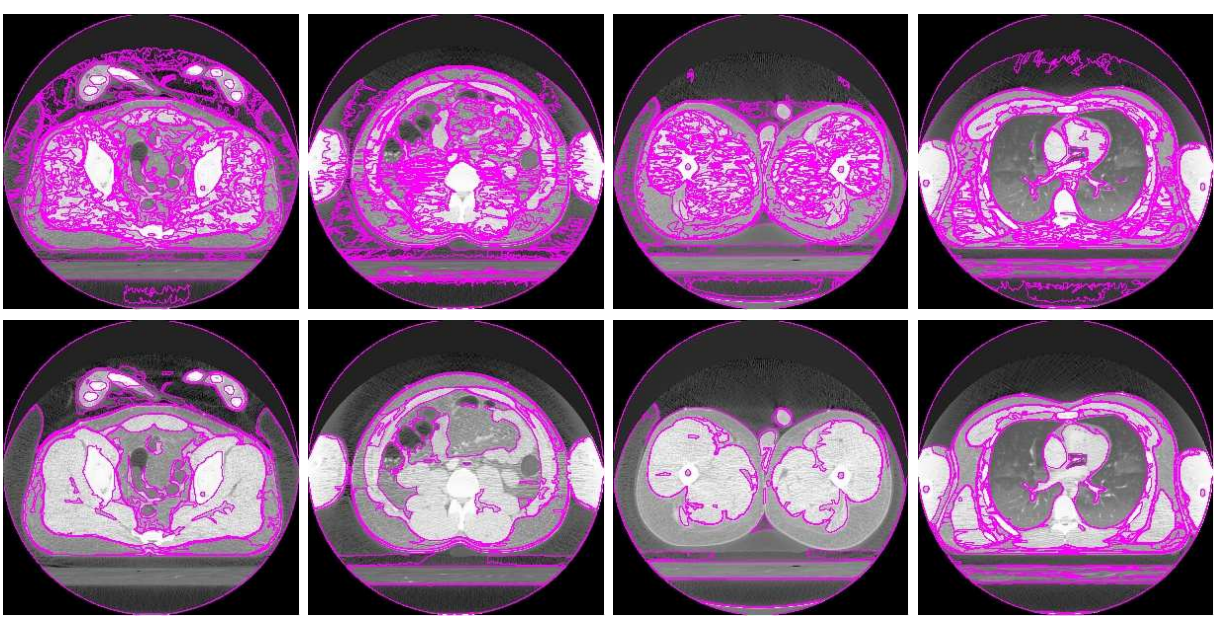

Fig. 8. First row: closed edge detection with the proposed selection principle prior to the curvature analysis. Second row: final boundaries set using curvature analysis.

8. A. Martelli, "Edge detection using heuristic search methods," Comp. Graph. and Image Proc., vol. 1, pp. 169-182, 1972.

9. U. Montanari, "On the optimal detection of curves in noisy images," Com. ACM, vol. 14, pp. 335-345, 1971.

10. G. Giraudon, "Chainage efficace des contours," Tech. Rep. 605, INRIA, 1987.

11. M. Kass, A. Witkin, and D. Terzopoulos, "Active contour models," Int. J. Comp. Vision, vol. 1, pp. 321-331, 1988.

12. A. Blake and A. Zisserman, Active Contours. Springer Verlag, 1998.

13. S. Osher and J. Sethian, "Front propagating with curvature dependant speed: algorithm based on the hamilton-jacobi formulations," Journal of Computational Physics, vol. 79, pp. 12-49, 1988.

14. J. Sethian, Level-set methods. Princeton University Press, 1996.

15. V. Caselles, R. Kimmel, and G. Sapiro, "Geodesic active contours," Int. J. Comput. Vision, vol. 22, pp. 61-79, 1997.

16. J. Serra, Image Analysis and Mathematical Morphology. Academic Press, 1982.

17. V. Caselles, B. Coll, and J.-M. Morel, "Topographic maps and local contrast changes in natural images," International Journal of Computer Vision, vol. 33, pp. 5-27, 1999.

18. A. Desolneux, L. Moisan, and J. Morel, "Edge detection by helmohltz principle," International Journal of Computer Vision, vol. 14, pp. 271-284, 2001.

19. F. Cao, P. Mus, and F. Sur, "Extracting meaningful curves from images," Tech. Rep. 5067, INRIA, Dec. 2003.

20. J. Duncan and N. Ayache, "Medical image analysis: progress over two decades and the challenges ahead," IEEE Trans. Pattern Anal. Machine Intell., vol. 22, pp. $85-106,2000$.

21. A. Desolneux, L. Moisan, and J.-M. Morel, "Meaningful alignments," International Journal of Computer Vision, vol. 40, pp. 7-23, 2000. 
22. A. Almansa, A. Desolneux, and S. Vamech, "Vanishing points detection without any a priori information," IEEE Trans. on Pattern Analysis and Machine Intelligence, vol. 25, pp. 502-507, april 2003.

23. A. Desolneux, L. Moisan, and J.-M. Morel, "A grouping principle and four applications," IEEE Trans. on Pattern Analysis and Machine Intelligence, vol. 25, no. 4, pp. 508-513, 2003.

24. P. Musé, F. Sur, F. Cao, and Y. Gousseau, "Unsupervised thresholds for shape matching," in Proceedings of the IEEE International Conference on Image Processing, (Barcelona, Spain), pp. 647-650, 2003.

25. T. Hurtut, H. Dalazoana, Y. Gousseau, and F. Schmitt, "Spatial color image retrieval without segmentation using thumbnails and the earth mover's distance." CGIV, Leeds, England, june 2006.

26. M. Murphey, J. Quale, N. Martin, J. Bramble, L. Cook, and S. Dwyer, "Computed radiography in musculoskeletal imaging: state of the art," American Journal of Roentgenology, vol. 158, no. 1, pp. 19-27, 1992.

27. A. Pizurica, A. Wink, E. Vansteenkiste, W. Philips, and J. BTM, "A Review of Wavelet Denoising in MRI and Ultrasound Brain Imaging," Current Medical Imaging Reviews, vol. 2, pp. 247-260, 2006.

28. J. Scharcanski, C. Jung, and R. Clarke, "Adaptive image denoising using scale and space consistency," Image Processing, IEEE Transactions on, vol. 11, no. 9, pp. 1092-1101, 2002.

29. J. Lee, "Digital image enhancement and noise filtering by use of local statistics," IEEE Transactions on Pattern Analysis and Machine Intelligence, 1980.

30. S. Kottamasu and L. Kuhns, "Musculoskeletal computed radiography in children: scatter reduction and improvement in bony trabecular sharpness using air gap placement of the imaging plate," Pediatric Radiology, vol. 27, no. 2, pp. 119-123, 1997.

31. R. Banvard, "The Visible Human Project@ Image Data Set From Inception to Completion and Beyond," Proceedings CODATA, 2002. 